\title{
Fachinales de Prosopis caldenia intervenidos por distintos manejos: Análisis desde su banco de semillas
}

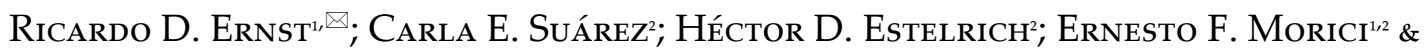 \\ Marilina A. Campos ${ }^{1}$ \\ ${ }^{1}$ Facultad de Ciencias Exactas y Naturales. Universidad Nacional de La Pampa. ${ }^{2}$ Facultad de Agronomía. Universidad \\ Nacional de La Pampa.
}

\begin{abstract}
Resumen. En los pastizales de la región semiárida central de la Argentina se observa un aumento de leñosas y de especies de bajo valor forrajero. Su recuperación como sitios de pastoreo depende del banco de semillas, de su composición y su dinámica ante distintas intervenciones. El objetivo fue determinar la composición y la abundancia de especies del banco de semillas del suelo germinable (BSSG) y su relación con la comunidad vegetal en distintos parches de vegetación de un área de renoval-fachinal de caldén intervenida con distintas técnicas de manejo. El trabajo se desarrolló en un ambiente con una cobertura de leñosas superior a $75 \%$. Mediante un rolado selectivo y una quema controlada se establecieron cuatro tratamientos: testigo $(\mathrm{T})$, quemado $(\mathrm{Q})$, rolado (R) y rolado+quemado $(R+Q)$. En cada uno se tomaron muestras de suelo en parches abiertos (predominio de gramíneas-herbáceas) y cerrados (con un estrato-arbóreo arbustivo) para estudiar el BSSG y se evaluó la abundancia-cobertura de la vegetación. Los datos se analizaron mediante los índices de similitud de Jaccard y Raup-Crick, NMDS, ANOVA, clúster a dos vías y PerMANOVA. En el BSSG se identificaron 34 especies y en la comunidad vegetal se registraron 61 especies. El 27\% de las especies del BSSG fueron gramíneas perennes, la mayoría forrajeras. La similitud entre el BSSG y la vegetación, entre tratamientos y parches de vegetación, fue baja. Sin embargo, el BSSG tuvo una similitud alta entre todos los tratamientos. Las gramíneas perennes forrajeras tuvieron mayor densidad de plántulas en los parches abiertos. Con respecto a los tratamientos, se observó menor densidad en T y mayor en los tratamientos disturbados. Las gramíneas no forrajeras perennes y las gramíneas y dicotiledóneas anuales emergieron más en los parches cerrados. Las especies exóticas aumentaron con los disturbios y tuvieron los mayores valores en R. En estos sistemas sometidos a variados disturbios, los parches de vegetación generan una heterogeneidad espacial que actúa como factor determinante de la composición y la dinámica del BSSG. En consecuencia, los parches de vegetación y el banco de semillas del suelo, como memoria de las comunidades, constituyen elementos a tener en cuenta para restaurar estos sistemas.
\end{abstract}

[Palabras clave: restauración, lignificación, disturbios, parches, BSSG]

Aвstract. Prosopis caldenia encroachments intervened by different management techniques: Analysis from its seedbank. Argentina's central semiarid area is characterized by an important increase of woody species and non-palatable grasses. The recovery of its grazing sites depends on the seed bank composition and dynamics under different management practices. The aim of this study was to analyse the composition and abundance of viable seed bank (BSSG) and its relationship with the plant community in different patches of vegetation in a caldén encroachment under different management practices. This work was carried out in a degraded caldén woodland with a woody cover greater than $75 \%$. After a selective rolling and a controlled burning, four treatments were obtained: control $(T)$, burned $(Q)$, rolled $(R)$ and rolled+burned $(R+Q)$. At each treatment site, in open patches (with a predominance of grasses and herbaceous plants) and closed ones (with a woody layer) vegetation cover was evaluated and soil samples were taken for GSSB analysis. Data were analysed using the Jaccard and Raup-Crick similarity index, NMDS, ANOVA, two-way cluster and PerMANOVA. Sixtyone species were recorded in the plant community and a 34 species were identified in the GSSB. The $26.5 \%$ of GSSB species were perennial grasses, mostly forage grasses (GFP). The similarity between the vegetation-BSSG in each treatment was low. However, the BSSG had a high similarity between all the treatments. Treatment patches had higher GFP seedling density than the Control. GFP seedling density was higher in open patches. Non-forage perennial grasses (GNFP) and GA+DA had higher seedling density in close patches. Exotic species density was higher in the treatment sites than in the Control, reaching higher values in the R site. In managed sites, heterogeneity in vegetation patches determines vegetation composition and GSSB dynamics. Therefore, the soil seed bank as a memory of the communities and vegetation patches are key elements to consider for the restoration of these systems.

[Keywords: restoration, lignification, disturbances, vegetation patches, GSSB]

Editora asociada: Marina Omacini $\bar{\triangle}$ ricardodanielernst@gmail.com
Recibido: 24 de Junio de 2019

Aceptado: 14 de Junio de 2020 


\section{INTRODUCCIÓN}

Los pastizales de regiones áridas y semiáridas manifestaron cambios importantes en su estructura y composición como consecuencia de los numerosos disturbios a los que fueron sometidos; entre ellos, la herbivoría y los incendios (Quiroga et al. 2009; Distel 2016). Una de las consecuencias más notorias de este deterioro fue el incremento de leñosas y de especies de bajo valor forrajero (Estelrich et al. 2005; Álvarez et al. 2012; Vásquez et al. 2013; De María et al. 2016). En la región semiárida central de la Argentina, este proceso de intensa degradación se inició con el ingreso del ganado doméstico (Bucher 1987; Archer 1995; Naito and Cairns 2011) y, actualmente, son frecuentes grandes áreas cubiertas por renovales y pajonales que, a su vez, coinciden con la ocurrencia de incendios (Estelrich et al. 2005).

La presencia y el aumento de la vegetación leñosa en estas comunidades genera condiciones ambientales diferenciales, con una disminución en la calidad y la cantidad de luz que llega al suelo, que repercute en la composición y la dinámica del pastizal (Leder et al. 2017; Álvarez Redondo and Adema 2018; Suárez et al. 2018). Estas condiciones, acentuadas por el pastoreo de herbívoros domésticos, constituyen fuerzas que seleccionan comunidades vegetales pobres desde el punto de vista de su diversidad florística y producción forrajera (Estelrich et al. 2005; Morici et al. 2009), cuya persistencia está muy relacionada con el banco de semillas del suelo (Harper 1977; Thompson and Grime 1979; Soriano 1990).

La restauración de estas áreas cubiertas por renovales-fachinales y pajonales, y su recuperación como sitios de pastoreo dependen del banco de semillas del suelo, de su composición y su dinámica frente a distintas intervenciones (Bedoya-Patiño et al. 2010; Kalamees et al. 2012). En este sentido, la expresión del BSS es el resultado de un proceso dinámico definido tanto por factores intrínsecos, condicionados por la estructura de la vegetación, como por factores extrínsecos; entre éstos, los disturbios son uno de los principales (Gianaccini et al. 2009; Morici et al. 2009; Bedoya-Patiño et al. 2010; Ernst et al. 2015; Ernst et al. 2017). En cuanto a los primeros, se demostró que los cambios en la calidad de luz, en la temperatura y en la humedad del suelo pueden provocar respuestas diferenciales en la germinación de las semillas (Fenner and Thompson 2005; Álvarez et al. 2012). Además, en cuanto a los segundos, algunos estudios muestran el efecto que los disturbios por pastoreo originan sobre la fecundidad de las especies forrajeras y las no forrajeras de los pastizales de la región (Estelrich et al. 2005; Morici et al. 2009). En las especies forrajeras, en general por cuestiones de manejo, se observan restricciones en la producción de semillas; y como consecuencia, un empobrecimiento en su participación relativa del BSS (Ernst et al. 2015; Martín et al. 2018; Ernst et al. 2017). Por el contrario, la presencia de las especies no forrajeras como las pajas, es altamente dominante, aun en parches de vegetación donde pudieran predominar forrajeras (Estelrich et al. 2005; Morici et al. 2009; Rauber et al. 2014).

El objetivo del presente trabajo fue estudiar el grado de asociación entre la composición del banco de semillas del suelo germinable (BSSG) y la composición de la comunidad establecida en distintos parches de vegetación de un área de renoval-fachinal de caldén intervenida con distintas técnicas de manejo. Simultáneamente, se buscó evaluar el impacto de la heterogeneidad espacial de la vegetación sobre la riqueza y composición del BSSG.

\section{Materiales y Métodos}

\section{Área de estudio}

El área de estudio se encuentra ubicada en el Establecimiento "Bajo Verde", campo experimental de la Universidad Nacional de La Pampa, Departamento Toay, provincia de La Pampa (36 $28^{\prime} 48^{\prime \prime}$ S - 64 $35^{\circ} 03^{\prime \prime}$ O; 235 m s. n. m.) (Figura 1). El trabajo se desarrolló en un potrero de 200 ha caracterizado por la presencia de un bosque degradado de caldén (Prosopis caldenia Burk.) cuya historia reciente de pastoreo consistió en la permanencia de ganado bovino de cría durante dos meses al año en otoño e invierno, con cargas moderadas (6-7 ha.UG ${ }^{-1} \cdot$ año $\left.^{-1}\right)$. Esta fisonomía, renovalfachinal, presentó una cobertura de leñosas superior a $75 \%$ con una altura variable del estrato de 1 a 3 metros. Parte de la superficie del potrero fue sometida a un rolado selectivo y a una posterior quema controlada.

\section{Diseño de los tratamientos}

Para definir los tratamientos consideraron las áreas del potrero sometidas a diferentes intervenciones (áreas roladas en el año 2013 y áreas quemadas en el año 2015). De esta 


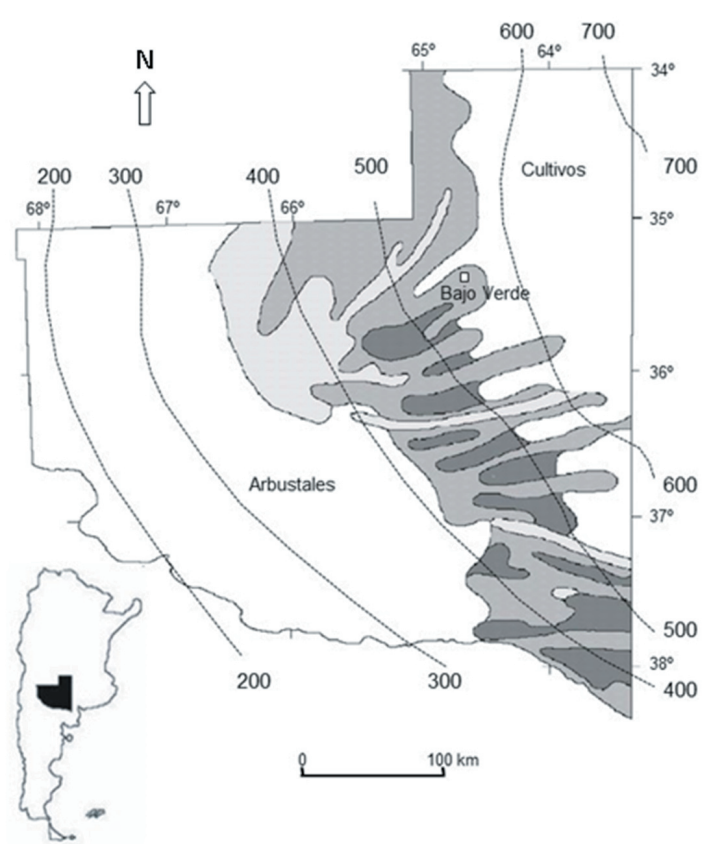

Figura 1. Ubicación del área de estudio. En gris intermedio: área con bosque de Prosopis caldenia; en gris claro: área de pastizal samófilo; en gris oscuro: área con pastizales bajos. Líneas punteadas: isohietas.

Figure 1. Location of the study area. In intermediate gray: Prosopis caldenia woodland area; in light gray: sandy grassland area; in dark gray: low grassland area. Dotted lines: isohyet.

manera, se obtuvieron cuatro situaciones de renoval-fachinal: testigo $(\mathrm{T})$, quemado $(\mathrm{Q})$, rolado $(\mathrm{R})$ y rolado+quemado $(\mathrm{R}+\mathrm{Q})$.

Para cada tratamiento se establecieron dos fajas de $100 \mathrm{~m}^{2}$, dentro de las cuales se tomaron muestras de suelo en dos situaciones contrastantes de cobertura de vegetación: parches abiertos (A) y cerrados (C) (20 muestras de suelo [10 en A y 10 en C] $x 4$ tratamientos; $n=20 ; N=80$ ). Los parches abiertos, sin presencia de leñosas, tuvieron un estrato graminoso-herbáceo con predominio de gramíneas forrajeras como Poa ligularis acompañada por Piptochaetium napostaense. Los parches cerrados presentaron un estrato arbóreo o arbustivo, con predominio de Prosopis caldenia acompañado por Condalia microphylla, Schinus johnstonii, Lyciun chilense y Ephedra triandra, y un estrato graminosoherbáceo donde predominaron especies no forrajeras como Nassella trichotoma, Nassella tenuissima y Jarava ichu.

\section{Extracción y análisis del banco de semillas del suelo}

El muestreo se realizó en marzo de 2018, luego de producida la lluvia de semillas de gramíneas y dicotiledóneas, mediante un cilindro de $7 \mathrm{~cm}$ de diámetro con el que se extrajeron los cuatro primeros centímetros del suelo, incluyendo la broza. Las muestras fueron secadas al aire $y$, posteriormente, se dispusieron en bandejas de germinación para registrar la emergencia de las plántulas, de acuerdo con el método de BSSG (Roberts 1981; Piudo and Cavero 2005). La experiencia se realizó en un invernadero automatizado con un período de luz de 12 horas diarias, a una temperatura aproximada de $10{ }^{\circ} \mathrm{C}$ por la noche y de $20^{\circ} \mathrm{C}$ durante el día. Las bandejas se regaron por primera vez a capacidad de campo y luego semanalmente según el requerimiento del sustrato.

Una vez producida la germinación de las semillas se contaron las plántulas que emergieron durante ocho meses (hasta que no se visualizó ninguna otra germinación). La identificación de plántulas se realizó durante los dos primeros meses en forma diaria, y los últimos meses en forma semanal (para más detalles ver Ernst et al. 2015). Para clasificar las especies en función de su calidad forrajera y no forrajera se tuvo en cuenta el grado de uso y preferencia de las mismas por parte del ganado bovino (Cano 1988; Rúgolo de Agrasar et al. 2005; Morici et al. 2009).

\section{Relevamiento de la vegetación}

Para registrar la presencia de la casi totalidad de especies que conforman la comunidad se realizaron censos florísticos (Mueller-Dombois and Ellemberg 1974; Braun-Blanquet 1979) siguiendo el diseño usado en el BSSG. Los muestreos se realizaron en diciembre y en marzo, en coincidencia con el período reproductivo.

\section{Análisis de datos}

Para evaluar la similitud entre el BSSG y la vegetación se utilizaron los índices de Jaccard y Raup- Crick, que se basan en datos de presencia-ausencia. El índice de Jaccard (Jaccard 1912) no hace ponderación y excluye las ausencias conjuntas (Magurran 1988; Rodríguez Zalazar et al. 2001; Kalacska et al. 2004; Hu et al. 2019). Por su parte, el índice de Raup-Crick (Raup and Crick 1979) usa una aleatorización (Monte Carlo) que compara el número observado de especies que ocurren en ambas asociaciones con la distribución de co-ocurrencias de 1000 repeticiones aleatorias del grupo de muestras (Raup and Crick 1979; Chase et al. 2011). Para ambos casos, la 
información fue presentada a través de un análisis de escalamiento multidimensional no métrico (NMDS) (Taguchi and Oono 2005) para representar la distribución de los distintos tratamientos en dos dimensiones, usando como medida de distancia la correspondiente a cada índice. Para testear las diferencias se utilizó la prueba de permutación ANOSIM (Clarke 1993). Por otra parte, los valores del índice de Raup-Crick se estandarizaron $\left(\beta_{\mathrm{RC}}\right)$ mediante la fórmula propuesta por Chase et al. (2011), en la cual se sustrae 0.5 al valor original obtenido y se multiplica por dos. Este análisis se utilizó para inferir cuál o cuáles son los eventos que explicarían la disimilitud entre las comunidades (Chase et al. 2011). Así, valores de $\beta_{\mathrm{RC}} \approx 0$ (Similitud $_{\text {observ }}$ ada $\approx$ Similitud esperada $_{\text {}}$ implican que los eventos estocásticos estructuran las comunidades con una elevada dispersión entre los ambientes; valores de $\beta_{\mathrm{RC}} \approx 1$ (Similitud observada $_{\text {Similitud }}$ perada) implican que los filtros ambientales de tipo determinísticas favorecen la disimilitud en la composición de especies, fuerzas bióticas o limitación en la dispersión conducen a la diferenciación en comunidades adyacentes; valores de $\beta_{\mathrm{RC}} \approx-1$ (Similitud $_{\text {observada }}>$ Similitu $\mathrm{d}_{\text {esperada }}$ ) implica que los filtros ambientales determinísticos compartidos generan mayor similitud entre los sitios como resultados de factores abióticos.

La densidad de plántulas se analizó siguiendo una estructura factorial con parcelas divididas, donde las parcelas principales fueron las intervenciones y las subparcelas, los parches abiertos y cerrados de vegetación. Las plántulas fueron agrupadas en gramíneas forrajeras perennes (GFP), gramíneas no forrajeras perennes (GNFP), gramíneas y dicotiledóneas anuales (GA+DA), exóticas (Ex).

Los datos fueron transformados como $W=\sqrt{P+1}$ (donde $P$ es la variable) para cumplir con el supuesto de homogeneidad de varianza (Sokal and Rohlf 1981). Para las diferencias entre las medias se usó la prueba de Tukey (a un nivel de confianza de 0.05).

Se realizó un análisis de clúster a dos vías con el método de Ward, y como medida de similitud, la distancia euclídea; para esto se construyó una matriz de covarianza de 80 censos x 13 especies. Las especies consideradas para este análisis fueron las que presentaron una frecuencia igual o superior al $50 \%$ en algunos de los tratamientos; de esta manera se mantuvo un balance apropiado de muestras y de variables (densidad por especie). Se aplicó la prueba de aleatorización PerMANOVA para establecer si existen diferencias en cuanto a la composición de especies entre los parches abiertos y cerrados de vegetación y los tratamientos. Todos los análisis y los gráficos se realizaron utilizando el paquete estadístico Infostat versión 2018 (Di Renzo et al. 2018), PCOrd 6 (McCune and Mefford 2011) y PAST ver. 3.26 (Hammer et al. 2001).

\section{Resultados}

En la comunidad vegetal se registraron 61 especies, comprendidas en 21 familias y representadas, en su mayoría, por Asteraceae y Poaceae (60\% en conjunto). El 59\% de las especies relevadas fueron de ciclo perenne y el $79 \%$ son consideradas no forrajeras (Tabla 1). En el BSSG se identificaron 34 especies, comprendidas en 13 familias entre las que también se destacaron las Asteraceae y Poaceae. El 27\% de las especies encontradas fueron gramíneas perennes, de las cuales el $65 \%$ fueron forrajeras. El 74\% de las especies restantes fueron gramíneas y dicotiledóneas anuales, entre las que se observaron un $35 \%$ de exóticas. Cabe destacar que no se registraron plántulas de leñosas en ninguno de los tratamientos.

La similitud en la composición de especies presentes en el BSSG y la vegetación, entre tratamientos y parches de vegetación, fue baja. El análisis NMDS mediante los índices de Jaccard y de Raup-Crick mostraron dos agrupamientos; por un lado, el BSSG y por otro, la vegetación (Jaccard: Eje1=0.75; Eje2=0.06; stress=0.16; ANOSIM: $P<0.001$; $R=0.999$; Raup-Crick: Eje1=0.73; Eje2=0.14; stress=0.15; ANOSIM: $P<0.001 ; R=0.94$ ) (Figura 2 a y b). Además, ambos índices también mostraron que el BSSG tuvo una alta similitud y no presentó diferencias entre tratamientos ni parches de vegetación (Jaccard: Eje1=0.62; Eje2=0.39; stress=0.08, ANOSIM: $P=0.33, R=0.09$; Raup-Crick: Eje1=0.43, Eje2=0.14, stress=0.10, ANOSIM: $P=0.25, R=0.25)$. Los valores del índice de Raup-Crick estandarizado $\left(\beta_{\mathrm{RC}}\right)$ para el BSSG y su correspondiente comunidad vegetal indicaron que en la estructuración de la comunidad intervienen no sólo efectos aleatorios ambientales (factores estocásticos) sino también filtros ecológicos (factores determinísticos) (predomino de valores de $\beta_{\mathrm{RC}} \approx 0$ y $\left.\beta_{\mathrm{RC}} \approx-1\right)$. Sin embargo, al analizar los valores del índice estandarizado para el BSSG o la comunidad vegetal de forma aislada la 
Tabla 1. Composición florística de la comunidad vegetal y del banco de semilla germinable, ciclo de vida y calidad forrajera de las especies. D: dicotiledóneas; M: monocotiledóneas; A: anuales; P: perennes; F: forrajera; NF: no forrajera. BSSG: banco de semillas germinable.

Table 1. Floristic composition of the plant community and the germinable seed bank, species life cycle and forage quality. D: dicotyledons; M: monocotyledonous; A: annual; P: perennial, F: forage; NF: no forage. BSSG: germinable seed bank.

\begin{tabular}{|c|c|c|c|c|c|c|}
\hline \multirow[t]{2}{*}{ Especies } & \multirow[t]{2}{*}{ Familia } & \multirow{2}{*}{$\begin{array}{c}\text { Clase } \\
\text { botánica }\end{array}$} & \multirow{2}{*}{$\begin{array}{c}\text { Ciclo de } \\
\text { Vida }\end{array}$} & \multirow{2}{*}{$\begin{array}{l}\text { Calidad } \\
\text { Forrajera }\end{array}$} & \multicolumn{2}{|c|}{ Presencia } \\
\hline & & & & & BSSG & Vegetación \\
\hline Acaena myriophylla & Rosaceae & $\mathrm{D}$ & $\mathrm{P}$ & NF & -- & $x$ \\
\hline Amelichloa brachychaeta & Poaceae & M & $\mathrm{P}$ & NF & -- & $\mathrm{x}$ \\
\hline Aphanes parodii & Rosaceae & $\mathrm{D}$ & A & NF & $\mathrm{x}$ & $\mathrm{x}$ \\
\hline Aristida pallens & Poaceae & $\mathrm{M}$ & $\mathrm{P}$ & NF & -- & $\mathrm{x}$ \\
\hline Austrobrickellia patens & Asteraceae & $\mathrm{D}$ & $\mathrm{P}$ & NF & -- & $\mathrm{x}$ \\
\hline Baccharis artemisioides & Asteraceae & $\mathrm{D}$ & $\mathrm{P}$ & NF & -- & $\mathrm{x}$ \\
\hline Baccharis crispa & Asteraceae & $\mathrm{D}$ & $\mathrm{P}$ & NF & -- & $\mathrm{x}$ \\
\hline Baccharis gilliesii & Asteraceae & $\mathrm{D}$ & $\mathrm{P}$ & NF & -- & $\mathrm{x}$ \\
\hline Baccharis glutinosa & Asteraceae & $\mathrm{D}$ & $\mathrm{P}$ & NF & -- & $\mathrm{x}$ \\
\hline Baccharis ulicina & Asteraceae & $\mathrm{D}$ & $\mathrm{P}$ & NF & -- & $\mathrm{x}$ \\
\hline Bowlesia incana & Apiaceae & $\mathrm{D}$ & A & NF & $\mathrm{x}$ & $\mathrm{x}$ \\
\hline Bromus catharticus & Poaceae & $\mathrm{M}$ & A & $\mathrm{F}$ & $\mathrm{x}$ & $\mathrm{x}$ \\
\hline Cardus thoermeri & Asteraceae & $\mathrm{D}$ & A & NF & $\mathrm{x}$ & $\mathrm{x}$ \\
\hline Cerastium glomeratum & Cariophilaceae & $\mathrm{D}$ & A & NF & $\mathrm{x}$ & -- \\
\hline Chascolytrum subaristatum & Poaceae & $\mathrm{M}$ & $\mathrm{P}$ & $\mathrm{F}$ & $\mathrm{x}$ & $\mathrm{x}$ \\
\hline Chenopodium album & Quenopodiaceae & $\mathrm{D}$ & A & NF & $\mathrm{x}$ & -- \\
\hline Condalia microphylla & Rhamnaceae & $\mathrm{D}$ & $\mathrm{P}$ & NF & -- & $\mathrm{x}$ \\
\hline Conyza blakei & Asteraceae & $\mathrm{D}$ & A & NF & $\mathrm{x}$ & $\mathrm{x}$ \\
\hline Cyclospermum leptophyllum & Apiaceae & $\mathrm{D}$ & A & NF & $\mathrm{x}$ & -- \\
\hline Daucus pucillus & Apiaceae & $\mathrm{D}$ & A & NF & $\mathrm{x}$ & $\mathrm{x}$ \\
\hline Descurainea erodiifolia & Brasicaceae & $\mathrm{D}$ & A & NF & $\mathrm{x}$ & $\mathrm{x}$ \\
\hline Dichondra microcalyx & Convolvulaceae & $\mathrm{D}$ & $\mathrm{P}$ & NF & -- & $\mathrm{x}$ \\
\hline Digitaria californica & Poaceae & $\mathrm{M}$ & $\mathrm{P}$ & $\mathrm{F}$ & $\mathrm{x}$ & $\mathrm{x}$ \\
\hline Erodium cicutarium & Geraniaceae & $\mathrm{D}$ & $\mathrm{P}$ & $\mathrm{F}$ & -- & $\mathrm{x}$ \\
\hline Facelis retusa & Asteraceae & $\mathrm{D}$ & A & NF & $\mathrm{x}$ & -- \\
\hline Galium richardianum & Rubiaceae & $\mathrm{D}$ & $\mathrm{P}$ & NF & -- & $\mathrm{x}$ \\
\hline Gaillardia megapotamica & Asteraceae & $\mathrm{D}$ & $\mathrm{P}$ & NF & -- & $\mathrm{x}$ \\
\hline Gamochaeta peregrina & Asteraceae & $\mathrm{D}$ & A & NF & $\mathrm{x}$ & -- \\
\hline Gamochaeta subfalcata & Asteraceae & $\mathrm{D}$ & A & NF & $\mathrm{x}$ & $\mathrm{x}$ \\
\hline Geranium molle & Geraniaceae & $\mathrm{D}$ & A & NF & $\mathrm{x}$ & -- \\
\hline Heterotheca subaxillaris & Asteraceae & $\mathrm{D}$ & A & NF & $\mathrm{x}$ & $\mathrm{x}$ \\
\hline Hipochaeris pampasica & Asteraceae & $\mathrm{D}$ & $\mathrm{P}$ & NF & -- & $x$ \\
\hline Hordeum stenostachys & Poaceae & $\mathrm{M}$ & A & NF & $\mathrm{x}$ & $\mathrm{x}$ \\
\hline Jarava ichu & Poaceae & M & $\mathrm{P}$ & NF & $\mathrm{x}$ & $\mathrm{x}$ \\
\hline Junellia hookeriana & Verbenaceae & $\mathrm{D}$ & $\mathrm{P}$ & NF & -- & $\mathrm{x}$ \\
\hline Lamium amplexicaule & Lamiaceae & $\mathrm{D}$ & A & NF & $\mathrm{x}$ & -- \\
\hline Lycium chilense & Solanaceae & $\mathrm{D}$ & $\mathrm{P}$ & NF & -- & $\mathrm{x}$ \\
\hline Lycium gilliesianum & Solanaceae & $\mathrm{D}$ & $\mathrm{P}$ & NF & -- & $\mathrm{x}$ \\
\hline Medicago minima & Fabaceae & $\mathrm{D}$ & A & $\mathrm{F}$ & $\mathrm{x}$ & $\mathrm{x}$ \\
\hline Nassella longiglumis & Poaceae & $\mathrm{M}$ & $\mathrm{P}$ & $\mathrm{F}$ & -- & $\mathrm{x}$ \\
\hline Nassella tenuis & Poaceae & $\mathrm{M}$ & $\mathrm{P}$ & $\mathrm{F}$ & -- & $\mathrm{x}$ \\
\hline Nassella tenuissima & Poaceae & $\mathrm{M}$ & $\mathrm{P}$ & NF & $\mathrm{x}$ & $\mathrm{x}$ \\
\hline Nassella trichotoma & Poaceae & $\mathrm{M}$ & $\mathrm{P}$ & NF & $\mathrm{x}$ & $\mathrm{x}$ \\
\hline Nierembergia aristata & Solanaceae & $\mathrm{D}$ & $\mathrm{P}$ & NF & -- & $\mathrm{x}$ \\
\hline Oenothera parodiana & Onagraceae & $\mathrm{D}$ & A & NF & $\mathrm{x}$ & -- \\
\hline Parietaria debilis & Urticaceae & $\mathrm{D}$ & A & NF & $\mathrm{x}$ & $\mathrm{x}$ \\
\hline Phalaris angusta & Poaceae & M & A & $\mathrm{F}$ & $\mathrm{x}$ & $\mathrm{x}$ \\
\hline Phaffia gnaphaloides & Amarantaceae & $\mathrm{D}$ & $\mathrm{P}$ & NF & -- & $\mathrm{x}$ \\
\hline Piptochaetium napostaense & Poaceae & M & $\mathrm{P}$ & $\mathrm{F}$ & $\mathrm{x}$ & $\mathrm{x}$ \\
\hline Plantago patagonica & Plantaginaceae & $\mathrm{D}$ & $\mathrm{A}$ & NF & $x$ & $x$ \\
\hline Poa ligularis & Poaceae & $\mathrm{M}$ & $\mathrm{P}$ & $\mathrm{F}$ & $\mathrm{x}$ & $\mathrm{x}$ \\
\hline Prosopis caldenia & Fabaceae & $\mathrm{D}$ & $\mathrm{P}$ & $\mathrm{F}$ & -- & $\mathrm{x}$ \\
\hline Pseudognaphalium gaudichaudianum & Asteraceae & $\mathrm{D}$ & A & NF & $\mathrm{x}$ & -- \\
\hline Salsola kali & Quenopodiaceae & $\mathrm{D}$ & $\mathrm{A}$ & NF & $x$ & $x$ \\
\hline Schinus fasciculatus & Ânacardiaceae & $\mathrm{D}$ & $\mathrm{P}$ & NF & -- & $\mathrm{x}$ \\
\hline Senecio subulatus & Asteraceae & $\mathrm{D}$ & $\mathrm{P}$ & NF & -- & $\mathrm{x}$ \\
\hline Setaria leucopila & Poaceae & $\mathrm{M}$ & $\mathrm{P}$ & $\mathrm{F}$ & $\mathrm{x}$ & -- \\
\hline Sonchus oleraceus & Asteraceae & $\mathrm{D}$ & A & NF & $\mathrm{x}$ & -- \\
\hline Sphaeralcea crispa & Malvaceae & $\mathrm{D}$ & $\mathrm{P}$ & NF & -- & $\mathrm{x}$ \\
\hline Sporobolus cryptandrus & Poaceae & M & $\mathrm{P}$ & $\mathrm{F}$ & $\mathrm{x}$ & $\mathrm{x}$ \\
\hline Taraxacum officinale & Asteraceae & $\mathrm{D}$ & $\mathrm{P}$ & NF & -- & $\mathrm{x}$ \\
\hline
\end{tabular}


mayor influencia está dada por los factores determinísticos (predominio de valores de $\beta_{\mathrm{RC}} \approx 1$ ) (Tabla 2).

A partir del análisis de la composición del BSSG según los distintos tratamientos, la mayor riqueza se observó en $\mathrm{R}$ y $\mathrm{R}+\mathrm{Q}$ con 28 y 31 especies, respectivamente (Tabla 3 ). Phalaris angusta tuvo la mayor densidad de plántulas en todas las intervenciones $y$, a su vez, los máximos valores se encontraron en los parches cerrados de vegetación del Q y del T. Heterotheca subaxillaris, una especie exótica, se destacó tanto en parches de vegetación cerrados como abiertos en los tratamientos $\mathrm{R}$ y R+Q. En estos últimos, también se observó una mayor densidad de Jarava ichu en los parches de vegetación cerrados mientras que Poa ligularis tuvo los mayores valores en las áreas abiertas intervenidas.

Al comparar la densidad de plántulas de las gramíneas forrajeras y no forrajeras perennes, y de las gramíneas y dicotiledóneas anuales, se observaron diferencias significativas entre los parches de vegetación, los tratamientos y la interacción entre ambos. En cuanto a las exóticas, un subgrupo dentro de las gramíneas y dicotiledóneas anuales, se observaron diferencias sólo entre los tratamientos (Figura
3). Las gramíneas forrajeras perennes tuvieron mayor densidad de plántulas en los parches de vegetación abiertos, y con respecto a los tratamientos, se observó menos cantidad en los testigos y aumentó en los disturbados. Se observaron diferencias significativas entre el $\mathrm{R}$ y $\mathrm{Q}$ con el T. En los parches de vegetación abiertos del $\mathrm{R}$ y del $\mathrm{Q}$ se registraron los mayores valores de estas especies (Figura 3a). Las gramíneas no forrajeras perennes tuvieron mayor densidad de plántulas emergidas en los parches de vegetación cerrados, principalmente en los tratamientos que involucraron el rolado (Figura 3b).

Las gramíneas y dicotiledóneas anuales también tuvieron mayor densidad de plántulas emergidas en los parches de vegetación cerrados, y con respecto a los tratamientos, si bien no hubo diferencias, se observó mayor densidad en los T y Q. La mayor cantidad de plántulas germinadas se observó en los parches de vegetación cerrados del Q con dominancia de Phalaris angusta (Figura 3c; Tabla 3). Dentro de este grupo, las exóticas estuvieron presente en todas las áreas, con diferencias entre los tratamientos $\mathrm{R}$ y $\mathrm{R}+\mathrm{Q}$ respecto al T. En el $\mathrm{R}$ se destacó Heterotheca subaxillaris por su alta densidad de plántulas (Figura 3d; Tabla 3). Con respecto a los parches

Tabla 2. Valores de similitud de Raup-Crick estandarizados $\left(\beta_{\mathrm{RC}}\right)$ según Chase et al. (2011) del banco de semillas germinable y de la vegetación provenientes de parches de vegetación abiertos y cerrados de sitios con diferentes tratamientos. Cuadros grises $=\beta_{\mathrm{RC}} \approx 0$ : Similitud observada $_{\text {Similitud }}$ esperada $($ entre $-0,5$ y 0,5$)$. Cuadros blancos $=\beta_{\mathrm{RC}} \approx 1$ :

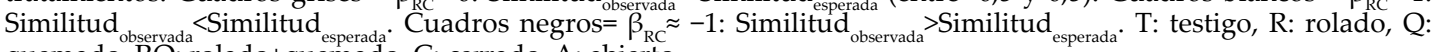
quemado, RQ: rolado+quemado, C: cerrado, A: abierto.

Table 2. Standardized Raup-Crick Similarity Values $\left(\beta_{\mathrm{RC}}\right)$ according to Chase et al. (2011) of the germinable seed bank and the vegetation from open and closed vegetation patches of sites with different treatments. Gray squares $=\beta_{\mathrm{RC}}$ $\approx 0$ : Similarity observed $\approx$ Similarities expected (between -0.5 and 0.5 ). White squares $=\beta_{\mathrm{RC}} \approx 1$ : Similarity observed $<$ Similarities expected. Black squares $=\beta_{\mathrm{RC}} \approx-1$ : Similarity observed $>$ Similarities expected. T: control, Q: burned, R: rolled and RQ: rolled+burned, C: closed, A: open.

\begin{tabular}{|c|c|c|c|c|c|c|c|c|c|c|c|c|c|c|c|c|c|}
\hline & & \multicolumn{8}{|c|}{ Banco de semillas germinable } & \multicolumn{8}{|c|}{ Vegetación } \\
\hline & & $\mathrm{T}_{\mathrm{A}}$ & $\mathrm{T}_{\mathrm{C}}$ & $\mathrm{R}_{\mathrm{A}}$ & $\mathrm{R}_{\mathrm{C}}$ & $\mathrm{Q}_{\mathrm{A}}$ & $\mathrm{Q}_{\mathrm{C}}$ & $\mathrm{RQ}_{\mathrm{A}}$ & $\mathrm{RQ}_{\mathrm{C}}$ & $\mathrm{T}_{\mathrm{A}}$ & $\mathrm{T}_{\mathrm{C}}$ & $\mathrm{R}_{\mathrm{A}}$ & $\mathrm{R}_{\mathrm{C}}$ & $\mathrm{Q}_{\mathrm{A}}$ & $Q_{C}$ & $\mathrm{RQ}_{\mathrm{A}}$ & $\mathrm{RQ}_{\mathrm{C}}$ \\
\hline \multirow{8}{*}{ 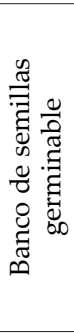 } & $\mathrm{T}_{\mathrm{A}}$ & & 1,00 & 1,00 & 1,00 & 0,99 & 1,00 & 0,94 & 1,00 & $-0,80$ & $-0,10$ & $-0,71$ & 0,94 & $-0,48$ & $-0,79$ & $-0,97$ & $-0,96$ \\
\hline & $\mathrm{T}_{\mathrm{C}}$ & - & & 1,00 & 1,00 & 0,94 & 1,00 & 0,99 & 1,00 & $-0,49$ & 0,41 & $-0,38$ & 0,90 & $-0,01$ & $-0,55$ & $-0,91$ & $-0,85$ \\
\hline & $\mathrm{R}_{\mathrm{A}}$ & - & - & & 1,00 & 1,00 & 1,00 & 1,00 & 1,00 & $-0,76$ & $-0,41$ & 0,21 & $-0,22$ & $-0,03$ & $-0,42$ & $-0,68$ & $-0,54$ \\
\hline & $\mathrm{R}_{\mathrm{C}}$ & - & - & - & & 0,97 & 1,00 & 1,00 & 1,00 & $-0,63$ & $-0,25$ & $-0,09$ & 0,13 & $-0,21$ & $-0,66$ & $-0,78$ & $-0,72$ \\
\hline & $\mathrm{Q}_{\mathrm{A}}$ & - & - & - & - & & 0,98 & 1,00 & 0,92 & $-0,45$ & $-0,58$ & $-0,50$ & 0,51 & $-0,48$ & $-0,14$ & $-0,71$ & $-0,65$ \\
\hline & $\mathrm{Q}_{\mathrm{C}}$ & - & - & - & - & - & & 0,98 & 1,00 & $-0,17$ & 0,69 & $-0,58$ & 0,56 & $-0,20$ & $-0,23$ & $-0,94$ & $-0,91$ \\
\hline & $\mathrm{RQ}_{\mathrm{A}}$ & - & - & - & - & - & - & & 1,00 & $-0,95$ & $-0,82$ & 0,45 & $-0,47$ & $-0,57$ & $-0,85$ & $-0,06$ & 0,17 \\
\hline & $\mathrm{RQ}_{\mathrm{C}}$ & - & - & - & - & - & - & - & & $-0,90$ & $-0,72$ & 0,29 & 0,17 & $-0,34$ & $-0,71$ & $-0,59$ & $-0,45$ \\
\hline \multirow{8}{*}{ 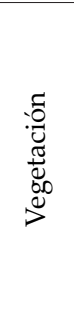 } & $\mathrm{T}_{\mathrm{A}}$ & - & - & - & - & - & - & - & - & & 0,78 & 0,97 & 0,78 & 0,96 & 0,96 & 0,40 & $-0,15$ \\
\hline & $\mathrm{T}_{\mathrm{C}}$ & - & - & - & - & - & - & - & - & - & & 0,58 & 0,78 & 0,93 & 0,98 & $-0,19$ & 0,48 \\
\hline & $\mathrm{R}_{\mathrm{A}}$ & - & - & - & - & - & - & - & - & - & - & & 0,82 & 1,00 & 0,93 & 0,99 & 0,97 \\
\hline & $\mathrm{R}_{\mathrm{C}}$ & - & - & - & - & - & - & - & - & - & - & - & & 0,81 & 0,94 & 0,14 & 0,65 \\
\hline & $\mathrm{Q}_{\mathrm{A}}$ & - & - & - & - & - & - & - & - & - & - & - & - & & 0,48 & 0,91 & 0,61 \\
\hline & $\mathrm{Q}_{\mathrm{C}}$ & - & - & - & - & - & - & - & - & - & - & - & - & - & & 0,30 & 0,77 \\
\hline & $\mathrm{RQ}_{\mathrm{A}}$ & - & - & - & - & - & - & - & - & - & - & - & - & - & - & & 1,00 \\
\hline & $R Q_{C}$ & - & - & - & - & - & - & - & - & - & - & - & - & - & - & - & \\
\hline
\end{tabular}


Tabla 3. Densidad (plántulas $/ \mathrm{m}^{2}$ ) y frecuencia (\%) de las especies presentes en el BSSG según los tratamientos y parches de vegetación. GFP: gramíneas forrajeras perennes; GFNP: gramíneas no forrajeras perennes; GA+DA: gramíneas y dicotiledóneas anuales; D: densidad; F: frecuencia; C: cerrado; A: abierto; Ex: exóticas. ${ }^{*}$ Especies utilizadas para los análisis multivariados.

Table 3. Density (seedlings $/ \mathrm{m}^{2}$ ) and frequency (\%) of the species present in the BSSG according to treatments and vegetation patches. GFP: perennial forage grasses; GFNP: perennial non-forage grasses; GA+DA: annual grasses and annual dicotyledons; D: density; F: frequency; C: closed; A: open; Ex: exotic. *Species used for multivariate analyses.

\begin{tabular}{|c|c|c|c|c|c|c|c|c|c|c|c|c|c|c|c|c|c|}
\hline \multirow{3}{*}{$\begin{array}{l}\text { Grupo } \\
\text { de } \\
\text { especies }\end{array}$} & \multirow{3}{*}{ Especies } & \multicolumn{4}{|c|}{ Testigo } & \multicolumn{4}{|c|}{ Quemado } & \multicolumn{4}{|c|}{ Rolado } & \multicolumn{4}{|c|}{ Rolado-Quemado } \\
\hline & & \multicolumn{2}{|c|}{$\mathrm{D}$} & \multicolumn{2}{|c|}{$\mathrm{F}$} & \multicolumn{2}{|c|}{$\mathrm{D}$} & \multicolumn{2}{|c|}{$\mathrm{F}$} & \multicolumn{2}{|c|}{$\mathrm{D}$} & \multicolumn{2}{|c|}{$\mathrm{F}$} & \multicolumn{2}{|c|}{$\mathrm{D}$} & \multicolumn{2}{|c|}{$\mathrm{F}$} \\
\hline & & $\mathrm{C}$ & A & $\mathrm{C}$ & $\mathrm{A}$ & $\mathrm{C}$ & $\mathrm{A}$ & $\mathrm{C}$ & A & $\mathrm{C}$ & A & $\mathrm{C}$ & A & $\mathrm{C}$ & A & $\mathrm{C}$ & $\mathrm{A}$ \\
\hline \multirow[t]{7}{*}{ GFP } & $\begin{array}{l}\text { Chascolytrum } \\
\text { subaristatum }\end{array}$ & 0 & 0 & 0 & 0 & 26 & 0 & 10 & 0 & 947 & 1158 & 40 & 50 & 816 & 0 & 50 & 0 \\
\hline & $\begin{array}{l}\text { Digitaria } \\
\text { californica }\end{array}$ & 26 & 0 & 10 & 0 & 0 & 0 & 0 & 0 & 0 & 26 & 0 & 10 & 26 & 26 & 10 & 10 \\
\hline & $\begin{array}{l}\text { Piptochaetium } \\
\text { napostaense }^{*}\end{array}$ & 26 & 184 & 10 & 30 & 0 & 1053 & 0 & 100 & 79 & 1868 & 30 & 90 & 211 & 1526 & 40 & 70 \\
\hline & Poa ligularis* & 474 & 2184 & 30 & 40 & 3474 & 11158 & 50 & 90 & 1421 & 22368 & 70 & 100 & 1053 & 4868 & 90 & 100 \\
\hline & Setaria leucopila & 0 & 0 & 0 & 0 & 26 & 0 & 10 & 0 & 26 & 105 & 10 & 20 & 0 & 0 & 0 & 0 \\
\hline & $\begin{array}{l}\text { Sporobolus } \\
\text { cryptandrus }\end{array}$ & 0 & 0 & 0 & 0 & 0 & 0 & 0 & 0 & 0 & 0 & 0 & 0 & 53 & 0 & 20 & 0 \\
\hline & Total & 526 & 2368 & & & 3526 & 12211 & & & 2474 & 25526 & & & 2158 & 6421 & & \\
\hline \multirow[t]{4}{*}{ GNFP } & Jarava ich $u^{*}$ & 2079 & 1368 & 90 & 80 & 3079 & 947 & 100 & 60 & 15553 & 3289 & 100 & 100 & 10579 & 1211 & 80 & 100 \\
\hline & $\begin{array}{l}\text { Nassella } \\
\text { tenuissima* }\end{array}$ & 289 & 105 & 50 & 20 & 421 & 0 & 60 & 0 & 5289 & 2474 & 100 & 90 & 5158 & 1474 & 100 & 70 \\
\hline & Nassella trichotoma & 0 & 79 & 0 & 20 & 0 & 0 & 0 & 0 & 553 & 605 & 50 & 60 & 342 & 1289 & 50 & 50 \\
\hline & Total & 2368 & 1553 & & & 3500 & 947 & & & 21395 & 6368 & & & 16079 & 3974 & & \\
\hline \multirow[t]{26}{*}{$\mathrm{GA}+\mathrm{DA}$} & Aphanes parodii & 0 & 5868 & 0 & 70 & 132 & 737 & 10 & 60 & 0 & 79 & 0 & 10 & 0 & 158 & 0 & 30 \\
\hline & Bowlesia incana & 237 & 158 & 30 & 40 & 711 & 53 & 50 & 10 & 132 & 26 & 20 & 10 & 0 & 132 & 0 & 10 \\
\hline & $\begin{array}{l}\text { Bromus } \\
\text { catharticus* }\end{array}$ & 4921 & 553 & 100 & 90 & 4737 & 3816 & 90 & 100 & 1868 & 1132 & 90 & 60 & 1316 & 1526 & 60 & 90 \\
\hline & $\begin{array}{l}\text { Cardus thoermeri } \\
\text { Ex }\end{array}$ & 0 & 132 & 0 & 20 & 0 & 0 & 0 & 0 & 105 & 26 & 30 & 10 & 263 & 53 & 30 & 20 \\
\hline & $\begin{array}{l}\text { Cerastium } \\
\text { glomeratum* }\end{array}$ & 526 & 816 & 50 & 60 & 2289 & 868 & 70 & 70 & 4737 & 5158 & 90 & 90 & 5026 & 1421 & 80 & 60 \\
\hline & $\begin{array}{l}\text { Chenopodium } \\
\text { album* Ex }\end{array}$ & 3184 & 684 & 100 & 100 & 2579 & 1579 & 90 & 90 & 2132 & 842 & 100 & 60 & 3053 & 763 & 100 & 80 \\
\hline & Conyza blakei & 0 & 0 & 0 & 0 & 0 & 0 & 0 & 0 & 263 & 0 & 10 & 0 & 868 & 0 & 20 & 0 \\
\hline & $\begin{array}{l}\text { Cyclospermum } \\
\text { leptophyllum }\end{array}$ & 0 & 0 & 0 & 0 & 158 & 0 & 20 & 0 & 0 & 79 & 0 & 10 & 26 & 26 & 10 & 10 \\
\hline & Daucus pusillus & 763 & 500 & 50 & 60 & 158 & 132 & 0 & 40 & 263 & 1237 & 50 & 80 & 368 & 1526 & 50 & 100 \\
\hline & $\begin{array}{l}\text { Descurainea } \\
\text { erodiifolia }\end{array}$ & 3184 & 1447 & 70 & 70 & 3447 & 0 & 90 & 0 & 658 & 289 & 70 & 30 & 1816 & 184 & 70 & 40 \\
\hline & Facelis retusa & 0 & 0 & 0 & 0 & 0 & 0 & 0 & 0 & 0 & 0 & 0 & 0 & 0 & 79 & 0 & 30 \\
\hline & $\begin{array}{l}\text { Gamochaeta } \\
\text { peregrina }\end{array}$ & 105 & 0 & 20 & 0 & 0 & 26 & 0 & 10 & 53 & 26 & 20 & 10 & 0 & 0 & 0 & 0 \\
\hline & $\begin{array}{l}\text { Gamochaeta } \\
\text { subfalcata* }\end{array}$ & 1421 & 605 & 70 & 40 & 1895 & 79 & 50 & 20 & 3605 & 4237 & 60 & 100 & 2421 & 2184 & 80 & 70 \\
\hline & Geranium molle Ex & 105 & 211 & 30 & 20 & 211 & 0 & 20 & 0 & 0 & 0 & 0 & 0 & 26 & 0 & 10 & 0 \\
\hline & $\begin{array}{l}\text { Heterotheca } \\
\text { subaxillaris* Ex }\end{array}$ & 1895 & 605 & 70 & 60 & 2553 & 184 & 90 & 50 & 6763 & 5395 & 90 & 100 & 6184 & 2447 & 90 & 90 \\
\hline & $\begin{array}{l}\text { Hordeum } \\
\text { stenostachys }\end{array}$ & 368 & 368 & 40 & 30 & 474 & 211 & 50 & 20 & 0 & 0 & 0 & 0 & 53 & 0 & 20 & 0 \\
\hline & $\begin{array}{l}\text { Lamium } \\
\text { amplexicaule Ex }\end{array}$ & 0 & 0 & 0 & 0 & 0 & 0 & 0 & 0 & 0 & 26 & 0 & 10 & 0 & 289 & 0 & 20 \\
\hline & $\begin{array}{l}\text { Medicago minima } \\
\text { Ex }\end{array}$ & 0 & 0 & 0 & 0 & 79 & 0 & 10 & 0 & 0 & 105 & 0 & 20 & 79 & 684 & 20 & 50 \\
\hline & $\begin{array}{l}\text { Oenothera } \\
\text { parodiana }\end{array}$ & 53 & 0 & 0 & 0 & 0 & 0 & 0 & 0 & 0 & 0 & 0 & 0 & 0 & 26 & 0 & 10 \\
\hline & Parietaria debilis & 1447 & 632 & 40 & 40 & 8553 & 289 & 100 & 40 & 4500 & 579 & 50 & 50 & 2447 & 395 & 80 & 40 \\
\hline & Phalaris angusta* & 19526 & 3026 & 100 & 100 & 32842 & 1605 & 90 & 50 & 895 & 289 & 50 & 30 & 237 & 0 & 40 & 0 \\
\hline & $\begin{array}{l}\text { Plantago } \\
\text { patagonica* }\end{array}$ & 0 & 105 & 0 & 20 & 0 & 974 & 0 & 90 & 26 & 1053 & 10 & 50 & 263 & 1263 & 50 & 80 \\
\hline & $\begin{array}{l}\text { Pseudognaphalium } \\
\text { gaudichaudianum }\end{array}$ & 526 & 316 & 30 & 20 & 132 & 0 & 10 & 0 & 105 & 211 & 20 & 20 & 0 & 184 & 0 & 20 \\
\hline & Salsola kali* Ex & 26 & 184 & 10 & 40 & 53 & 1711 & 10 & 70 & 26 & 2316 & 10 & 60 & 368 & 2289 & 30 & 90 \\
\hline & \multirow{2}{*}{$\begin{array}{l}\text { Sonchus oleraceus } \\
\text { Ex }\end{array}$} & 0 & 211 & 0 & 30 & 0 & 26 & 0 & 10 & 0 & 0 & 0 & 0 & 0 & 0 & 0 & 0 \\
\hline & & 38289 & 16421 & & & 61000 & 12289 & & & 26132 & 23105 & & & 24816 & 15632 & & \\
\hline
\end{tabular}



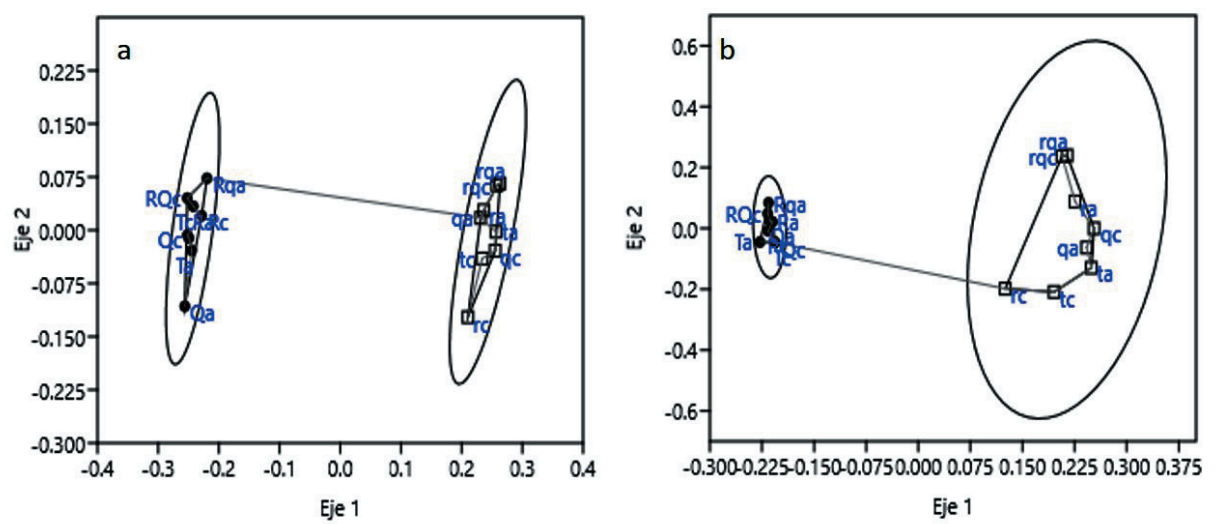

Figura 2. Análisis de escalamiento multidimensional no métrico (NMDS) a partir del índice de similitud de Jaccard (a) y el índice de Raup-Crick (b), de la composición del banco de semillas germinable y de la comunidad vegetal entre tratamientos y parches. Letras minúsculas indican la comunidad vegetal. Letras mayúsculas indican el banco de semillas germinable. T: testigo; R: rolado; Q: quemado; RQ: rolado+quemado; A: abierto; C: cerrado.

Figure 2. Non-metric multidimensional scale analyses (nMDS) using the Jaccard similarity index (a) and Raup-Crick index (b), the germinable seeds bank composition and the plant community, between treatments and patches of vegetation. Lowercase letters indicate the plant community. Capital letters indicate the germinable seed bank. T: control; Q: burned; R: rolled; RQ: rolled+burned; A: open; C: closed.
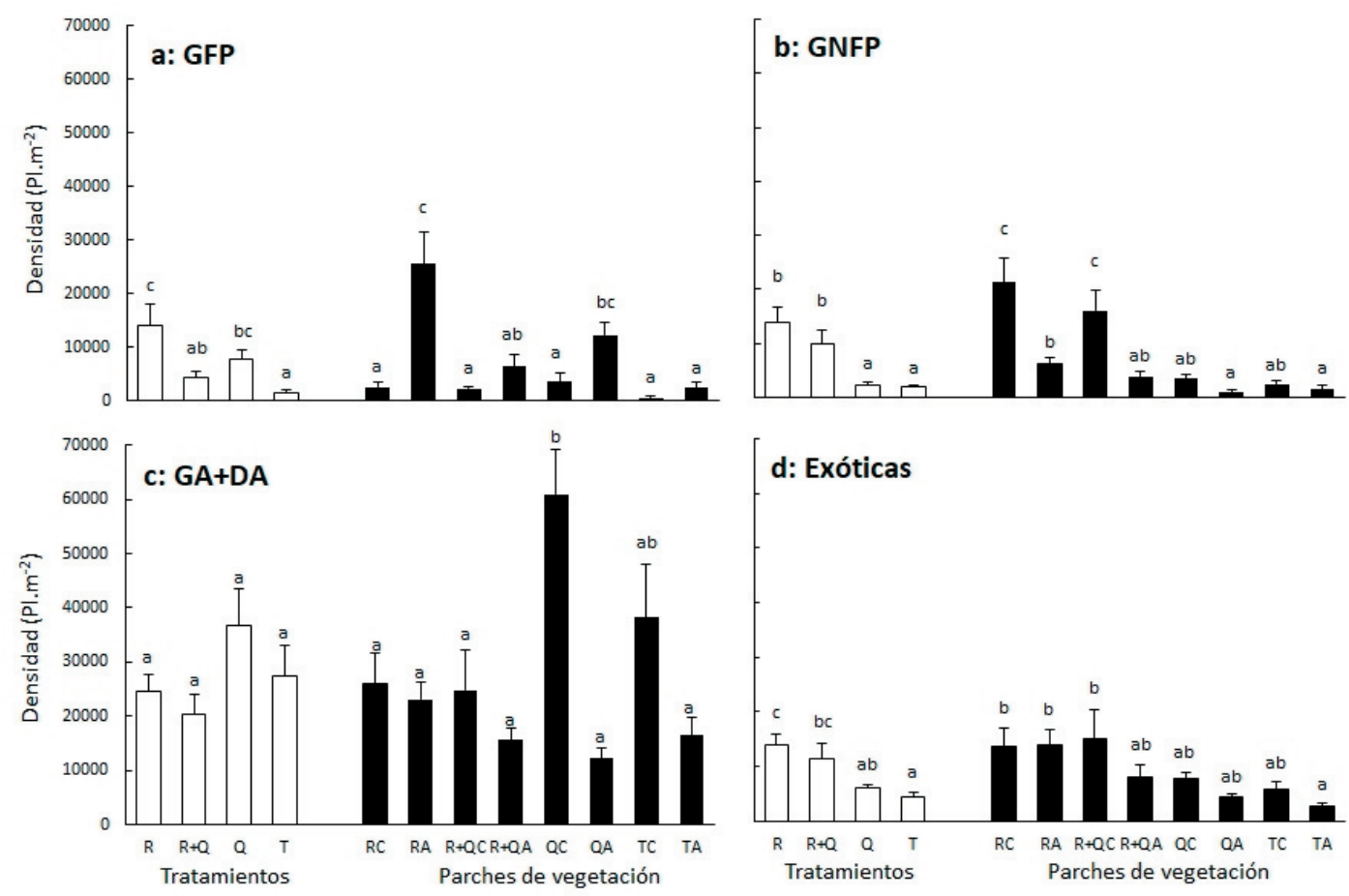

Figura 3. Densidad total (plántulas $/ \mathrm{m}^{2}$ ) de las especies presentes en el BSSG, analizadas por grupo de especies para cada tratamiento (R: rolado; R+Q: rolado+quemado; Q: quemado; T: testigo). A su vez, cada tratamiento se divide según el parche de vegetación (RC: rolado cerrado; RA: rolado abierto; R+QC: rolado+quemado cerrado; R+QA: rolado+quemado abierto; QC: quemado cerrado; QA: quemado abierto; TC: testigo cerrado; TA: testigo abierto). GFP: gramíneas forrajeras perennes; GNFP: gramíneas no forrajeras perennes; GA+DA: gramíneas anuales y Dicotiledóneas anuales. Letras distintas indican diferencias significativas $(P<0.05)$ entre los tratamientos (barras blancas). Letras distintas indican diferencias significativas $(P<0.05)$ entre los parches de vegetación de todos los tratamientos (barras negras).

Figure 3. Total density (seedlings $/ \mathrm{m}^{2}$ ) of the species present in the GSSB, analysed by group of species for each treatment and in turn (R: rolled; R+Q: rolled+burned; Q: burned; T: control). Each treatment is divided according to the vegetation patch (RC: closed rolling; RA: open rolling; R+QC: rolled+burned closed; R+QA: rolled+burned open; QC: burned closed; QA: burned open; TC: closed control; TA: open control). GFP: perennial forage grasses; GNFP: perennial non-forage grasses; GA+DA: annual grasses and annual dicotyledons. Different letters indicate significant differences $(P<0.05)$ between treatments (white bars). Different letters indicate significant differences $(P<0.05)$ between the vegetation patches of all the treatments (black bars). 
de vegetación, los cerrados tuvieron la mayor densidad de semillas germinadas.

A partir del análisis de clasificación a dos vías se identificaron tres agrupaciones (57.7\% de información para los tratamientos y $39.4 \%$ de información para las especies; porcentajes de encadenamiento=27.27; 13.11) (Figura 4). El grupo 1 estuvo integrado por los parches de vegetación abiertos de todos los tratamientos, caracterizado por Poa ligularis, que fue la más abundante, acompañada por Cerastium glomeratum, Gamochaeta subfalcata y Heterotheca subaxillaris. El grupo 2 estuvo conformado por los parches de vegetación cerrados de los tratamientos $\mathrm{R}$ y $\mathrm{R}+\mathrm{Q}$, con predominio de Jarava ichu, acompañada de Heterotheca subaxillaris, Cerastium glomeratum y Nassella tenuissima. Por último, el grupo 3 estuvo conformado por los parches de vegetación cerrados de los tratamientos Ty Q, caracterizado por Phalaris angusta, que se destacó por su alta densidad, y otras acompañantes como Bromus catharticus, Chenopoium album, Heterotheca subaxillaris y Gamochaeta subfalcata (Figura 4). En cuanto a la composición de especies del BSSG, el análisis de PerMANOVA mostró que hubo interacción entre los parches de vegetación y los tratamientos $(F=39.135, P=0.002)$.

\section{Discusión}

Numerosos trabajos mencionan la importancia del banco de semillas para restaurar las comunidades vegetales degradadas, con distintos tipos de manejo o intervenciones (Wang et al. 2010; Bertiller and Carrera 2015; Montoya et al. 2017; Hu et al. 2019). En este sentido, los trabajos que analizan la similitud entre banco de semillas y vegetación obtuvieron resultados muy dispares, independientemente de los índices utilizados (Márquez et al. 2002; Caballero et al. 2008; Kassahun et al. 2009; Montoya et al. 2017; Görzen et al. 2019; Hu et al. 2019), relacionados con los sistemas ecológicos y el grado de disturbio al que fueron sometidos (Wang et al. 2013).

En este trabajo se destacan dos aspectos importantes. Por un lado, la semejanza entre los BSSG y, por otro, la disimilitud entre

Figura 4. Análisis de clúster a dos vías. Distribución de los tratamientos y parches de vegetación estudiados en relación a las especies más representativas en el BSSG. TC: testigo cerrado; TA: testigo abierto; RC: rolado cerrado; RA: rolado abierto; R+QC: rolado+quemado cerrado; $\mathrm{R}+\mathrm{QA}$ : rolado+quemado abierto; QC: quemado cerrado; QA: quemado abierto. Pli: Poa ligularis; Pna: Piptochaetium napostaense; Jic: Jarava ichu; Ntss: Nassella tenuissima; Bca: Bromus catharticus; Cgl: Cerastium glomeratum; Chal: Chenopodium album; Gsu: Gamochaeta subfalcata; Gga: Pseudognaphalium gaudichaudianum; $\mathrm{Hsu}$ : Heterotheca subaxillaris; Pan: Phalaris angusta; Ppa: Plantago patagonica; Ska: Salsola kali.

Figure 4. Two-way cluster analysis. Distribution of the treatments and patches of vegetation studied in relation to the most representative species in the GSSB. TC: closed control; TA: open control; RC: closed rolling; RA: open rolling; R+QC: rolled+burned closed; R+QA: rolled+burned open; $\mathrm{QC}$ : burned closed; QA: burned open. Pli: Poa ligularis; Pna: Piptochaetium napostaense; Jic: Jarava ichu; Ntss: Nassella tenuissima; Bca: Bromus catharticus; Cgl: Cerastium glomeratum; Chal: Chenopodium album; Gsu: Gamochaeta subfalcata; Gga: Pseudognaphalium gaudichaudianum; Hsu: Heterotheca subaxillaris; Pan: Phalaris angusta; Ppa: Plantago patagonica; Ska: Salsola kali.

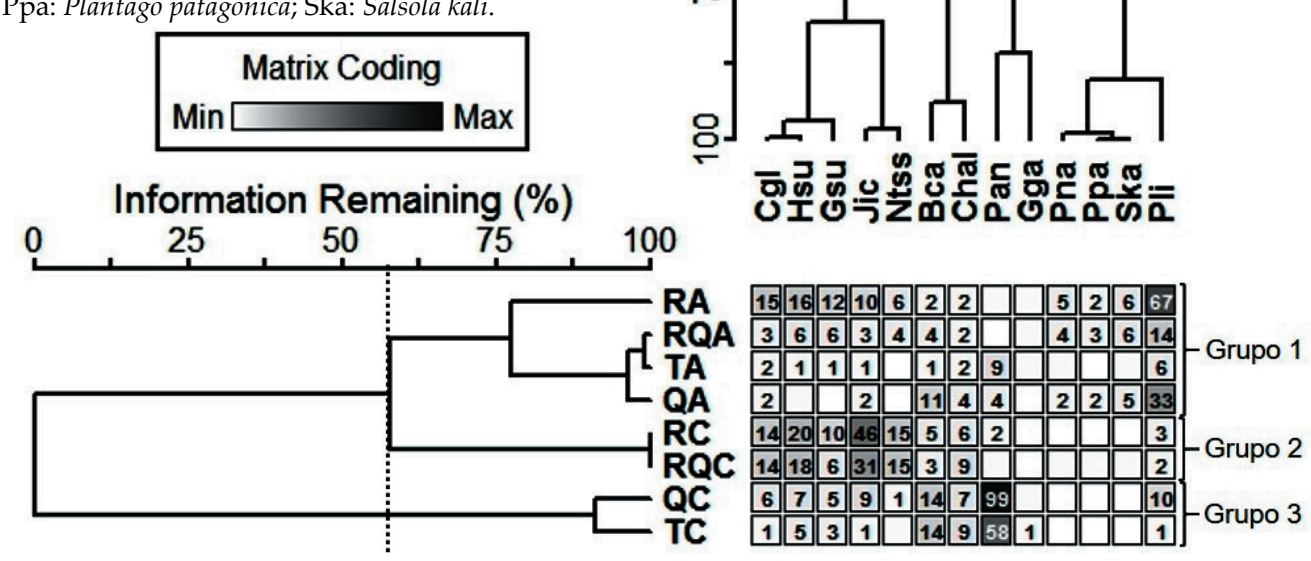


el BSSG y la comunidad vegetal. Así, la homogeneidad observada en la composición del BSSG a nivel de parches de vegetación se podría explicar por su tamaño reducido y por la estructura de la vegetación, más que por las especies individuales allí presentes. $\mathrm{Al}$ respecto, Caballero et al. (2008) mencionan que el efecto parche se extiende fuera de sus límites, creandoun gradiente en la composición y densidad del BSS. Esto podría provocar que los parches de vegetación abiertos y cerrados estén influenciados entre sí (Estelrich et al. 2005; Caballero et al. 2008; Morici et al. 2009; Leder et al. 2017). Mientras tanto, en la disimilitud observada entre la comunidad vegetal y el BSSG, un factor importante es la escala temporal que define a ambos, ya que las comunidades vegetales resultantes de las distintas intervenciones son más jóvenes que el banco de semillas (Hopfensperger 2007; Hu et al. 2019). Esto revela la importancia del rol del banco de semilla como 'memoria' de las comunidades (Templeton and Levin 1979; Marañon 2011; Hu et al. 2019).

Los parches de vegetación cerrados, más allá de los tratamientos, se caracterizaron por especies con características similares en sus semillas, que les permitieron concentrarse en estos lugares (i.e., gramíneas no forrajeras perennes, gramíneas y dicotiledóneas anuales). Estos grupos presentan semillas livianas y pequeñas, con estructuras de dispersión principalmente anemófilas (Rúgolo de Agrasar et al. 2005; Suárez et al. 2010; Suárez and Estelrich 2018), que son fácilmente interceptadas debido a la complejidad de la estructura vertical de los estratos inferiores de vegetación y broza (Caballero et al. 2008). Esto asegura la emergencia de plántulas en áreas con mayor cobertura y mejores condiciones de humedad y nutrientes (Noellemeyer et al. 2006; Gili et al. 2010; Suárez et al. 2018). Por otra parte, en los parches de vegetación abiertos, las mayores densidades de las gramíneas forrajeras perennes estarían asociadas a la historia reciente de pastoreo moderado ( $\mathrm{Hu}$ et al. 2019; Estelrich et al. 2005) y a la mayor cantidad de luz que llega al suelo (Ernst et al. 2017), ya que en condiciones de sombreado estas especies tendrían menor emergencia, crecimiento y supervivencia, junto con una menor tolerancia al pastoreo (Estelrich et al. 2005). En función de lo antes mencionado, los parches de vegetación abiertos tienen una composición diferente a los cerrados, y a su vez dentro de estos últimos, se observa una distinción entre los provenientes de áreas menos y más disturbadas.
Teniendo en cuenta los distintos tratamientos y las técnicas de manejo aplicadas, el incremento en la densidad de semillas germinadas de las gramíneas forrajeras y no forrajeras perennes observado en las áreas con intervención mecánica $(R$ y $R+Q)$ se explicaría a partir del efecto que provoca el rolado sobre el suelo. En las áreas roladas, esta acción origina un proceso de liberación competitiva del espacio por desarraigo y muerte de individuos (Dreber and Esler 2011), junto con un aumento del contenido de carbono orgánico y de nitrógeno total en los primeros centímetros del suelo (Somovilla Lumbrera et al. 2019). Además, observamos la formación de microcuencas sobre la superficie, que junto con la mayor llegada de luz (Suárez et al. 2018) favorecería el ingreso de semillas de estas especies al BSS y su establecimiento posterior.

En el mismo sentido, se observó que en todas las áreas intervenidas también aumentó la densidad de plántulas emergidas de las especies exóticas (Gilfedder and Kirkpatric 1993; Hu et al. 2019), con los máximos valores en el tratamiento rolado. Estos resultados ponen de manifiesto la vulnerabilidad del sistema a la invasión de estas especies (Lonsdale 1999; Davis et al. 2000; Huston 2004; Suárez et al. 2019) y coinciden con lo reportado por distintos autores para otros pastizales de Sudamérica (Fonseca et al. 2013) y del mundo (Foxcroft et al. 2010).

La ausencia de plántulas de leñosas en el BSSG se debería a la inmadurez del estrato de leñosas del sistema, dominado por ejemplares de caldén en estado pre-reproductivo que aún no alcanzaron la floración-fructificación. Además, no se detectó la presencia de ganado bovino que favorezca la diseminación desde otros potreros.

En este trabajo se identifica la heterogeneidad de la vegetación, definida a partir de los mosaicos de vegetación, como uno de los factores determinantes de la composición y abundancia del BSS, que funcionaría como la memoria delas comunidades vegetales. Ambos son aspectos fundamentales para el manejo o la restauración de estas áreas afectadas por distintas actividades antrópicas (Chaudhary et al. 2009; Golos and Dixon 2014).

Agradecimientos. El trabajo fue financiado por el programa POIRe de la UNLPam (Proyectos Orientados a la Investigación Regional), por el Proyecto de Investigación 
$N^{\circ} 37 / 2014$ "Efecto del Rolado Selectivo y la Quema Controlada sobre el Banco de Semillas" del Dpto. de Recursos Naturales, Facultad de Ciencias Exactas y Naturales UNLPam y por proyectos específicos de la Facultad de Agronomía, UNLPam. Se agradece la colaboración de Nicolás Parodi y Mauro
Pérez Payeras por las tareas de muestreo, al Dr. Alberto Pilati por las sugerencias realizadas y a la Dra. Patricia Torres por sus valiosos aportes y sugerencias en los análisis estadísticos. A su vez, se agradecen las valiosas sugerencias realizadas por los revisores anónimos de este trabajo.

\section{REFERENCIAS}

Álvarez Redondo, M., and E. Adema. 2018. Capacidad de retención de agua por el dosel de los arbustos en la región árida-semiárida central de Argentina. Semiárida. Revista de la Facultad de Agronomía. UNLPam 28(1):31-40. https: //doi.org/10.19137/semiarida.2018(01).3140.

Álvarez, L. J., H. E. Epstein, J. Li, and G. S. Okin. 2012. Aeolian process effects on vegetation communities in an arid grassland ecosystem. Ecology and Evolution 2(4):809-821. https://doi.org/10.1002/ece3.205.

Archer, S. 1995. Tree-grass dynamics in a Prosopis-thornscrub savanna parkland: Reconstructing the past and predicting the future. Ecoscience 2(l):83-99. https://doi.org/10.1080/11956860.1995.11682272.

Bedoya-Patiño, J. G., J. V. Estévez-Varón, and G. J. Castaño-Villa. 2010. Banco de semillas del suelo y su papel en la recuperación de los bosques tropicales. Boletín Científico de Museos de Historia Natural 14(2):77-91.

Bertiller, M. B., and A. L. Carrera. 2015. Aboveground Vegetation and Perennial Grass Seed Bank in Arid Rangelands Disturbed by Grazing. Rangeland Ecology and Management 68(1):71-78. https://doi.org/10.1016/j.rama.2014.12.008. Braun-Blanquet, J. 1979. Fitosociología. Bases para el estudio de las comunidades vegetales. Ed. Blume.

Bucher, E. H. 1987. Herbivoría en las regiones áridas y semiáridas de Argentina. Revista Chilena de Historia Natural 60:265-273.

Caballero, I., J. M. Olano, A. Escudero, and J. Loidi. 2008. Seed bank spatial structure in semi-arid environments: beyond the patch-bare area dichotomy. Plant Ecology 195:215-223. https://doi.org/10.1007/s11258-007-9316-7.

Cano, A. E. 1988. Pastizales naturales de La Pampa. Tomo I: Descripción de las especies más importantes. Convenio AACREA - Provincia de La Pampa. Zona semiárida. Pp. 456.

Chase, J. M., N. J. B. Kraft, K. G. Smith, M. Yellend, and B. D. Inouye. 2011. Using null models to disentangle variation in community dissimilarity from variation in a-diversity. Ecosphere 2(2):1-11. https://doi.org/10.1890/ES10-00117.1.

Chaudhary, V. B., M. A. Bowker, T. E. O’Dell, J. B. Grace, A. E. Redman, M. C. Rillig, and N. C. Johnson. 2009. Untangling the biological contributions to soil stability in semiarid shrublands. Ecological Applications 19(1):110-122. https: //doi.org/10.1890/07-2076.1.

Clarke, K. R. 1993. Non-parametric multivariate analysis of changes in community structure. Australian Journal of Ecology 18:117-143. https://doi.org/10.1111/j.1442-9993.1993.tb00438.x.

Davis, M. A., P. J. Grime, and K. Thompson. 2000. Fluctuating resources in plant communities: a general theory of invasibility. Journal of Ecology 88:528-534. https://doi.org/10.1046/j.1365-2745.2000.00473.x.

Demaría, M. R., J. P. Martini, and D. F. Steinaker. 2016. Actualización del límite occidental del pastizal pampeano. Ecología Austral 26:59-63. https://doi.org/10.25260/EA.16.26.1.0.141.

Di Rienzo, J. A., F. Casanoves, M. G. Balzarini, L. González, M. Tablada, and C. W. Robledo. 2018. Centro de Transferencia InfoStat, FCA, Universidad Nacional de Córdoba, Argentina.

Distel, R. A. 2016. Grazing ecology and the conservation of the Caldenal rangelands, Argentina. Journal of Arid Environment 134:49-55. https://doi.org/10.1016/j.jaridenv.2016.06.019.

Dreber, N., and K. J. Esler. 2011. Spatio-temporal variation in soil seed banks under contrasting grazing regimes following low and high seasonal rainfall in arid Namibia. Journal of Arid Environments 75:174-184. https://doi.org/ 10.1016/j.jaridenv.2010.09.007.

Ernst, R. D., E. Morici, H. D. Estelrich, W. A. Muiño, and M. A. Ruiz. 2015. Efecto de la quema controlada sobre el banco de semillas de gramíneas en diferentes parches del bosque de caldén en la región semiárida central Argentina. Archivos de Zootecnia 64(287):245-254. https://doi.org/10.21071/az.v64i247.404.

Ernst, R., V. Vásquez, D. Estelrich, and E. Morici. 2017. Banco de semillas de gramíneas en fachinales intervenidos mediante rolado selectivo. Semiárida. Revista de la Facultad de Agronomía. UNLPam 27(1):27-39. https://doi.org/ 10.19137/semiarida.2017(01).27-39.

Estelrich, H. D., C. C. Chirino, E. Morici, and B. Fernández. 2005. Dinámica de áreas naturales cubiertas por bosque y pastizal en la región semiárida central de Argentina - Modelo Conceptual. Pp.351-364 en J. Paruelo, M. Oesterheld and M. Aguiar (eds.). Heterogeneidad de la Vegetación. Libro homenaje a Rolando León. Facultad de Agronomía. Universidad de Buenos Aires.

Fenner, M., and K. Thompson. 2005. The ecology of seed. Cambridge University Press. United Kingdom.

Fonseca, C. R., D. L. Guadagnin, C. E. S. Masciadri, P. Germain, and S. M. Zalba. 2013. Invasive alien plants in the Pampas grasslands: a tri-national cooperation challenge. Biological Invasions 15:1751-1763. https://doi.org/10.1007/ s10530-013-0406-2.

Foxcroft, L. C., D. M. Richardson, M. Rejmánek, and P. Pyšek. 2010. Alien plant invasions in tropical and sub-tropical savannas: patterns, processes and prospects. Biological Invasions 12:3913-3933. https://doi.org/10.1007/s10530-010- 
9823-7.

Gianaccini, F., R. Scaramuzzino, and E. Requesens. 2009. Banco de semillas en ambientes de las sierras de Azul (Buenos Aires, Argentina) con distinta intensidad de disturbios. Agriscientia 26(2):71-79.

Gilfedder, L., and J. B. Kirkpatrick. 1993. Germinable soil seed and competitive relationships between a rare native species and exotics in a semi-natural pasture in the midlands. Tasmania. Biological Conservation 64:113-119. https: //doi.org/10.1016/0006-3207(93)90647-J.

Gili, A. A., R. Trucco, S. Niveyro, M. Balzarini. H. D. Estelrich, A. Quiroga, and E. Noellemeyer. 2010. Soil texture and carbon dynamics in savannah vegetation patches of Central Argentina. Forest, Range and Wildland Soils 74:647-657. https://doi.org/10.2136/sssaj2009.0053.

Golos, P. J., and K. W. Dixon. 2014. Waterproofing topsoil stockpiles minimizes viability decline in the soil seed bank in an arid environment. Restoration Ecology 22(4):495-501. https://doi.org/10.1111/rec.12090.

Görzen, E., K. Borisova, A. Fenesi, E. Ruprecht, and T. W. Donath. 2019. Effects of woody species encroachment and fire on vegetation and the soil seed bank in dry grasslands of Transylvania. Applied Vegetation Science 22:1-14. https: //doi.org/10.1111/avsc.12435.

Hammer, O., D. A. T. Harper, and P. D. Ryan. 2001. PAST: Paleontological statistics software package for education and data analysis. Palaeontologia Electronica 4(1):9pp.

Harper, J. L. 1977. Population Biology of Plants. London: Academic Press.

Hopfensperger, K. N. 2007. A review of similarity between seed bank and standing vegetation across ecosystems. Oikos 116:1438-1448. https://doi.org/10.1111/j.0030-1299.2007.15818.x.

Hu, A., J. Zhang, X. Chen, S. Chang, and F. Hou. 2019. Winter Grazing and Rainfall Synergistically Affect Soil Seed Bank in Semiarid Area. Rangeland. Ecology and Management 72:160-167. https://doi.org/10.1016/j.rama.2018.07.012.

Huston, M. A. 2004. Management strategies for plant invasions: manipulating productivity, disturbance, and competition. Diversity and Distributions 10:167-178. https://doi.org/10.1111/j.1366-9516.2004.00083.x.

Jaccard, P. 1912. The distribution of the flora of the alpine zone. New Phytology 11:37-50. https://doi.org/10.1111/j.14698137.1912.tb05611.x.

Kalacska, M., G. A. Sanchez-Azofeifa, J. C. Calvo-Alvarado, M. Quesada, B. Rivard, and D. H. Janzen. 2004. Species composition, similarity and diversity in three successional stages of a seasonally dry tropical forest. Forest Ecology and Management 200:227-247. https://doi.org/10.1016/j.foreco.2004.07.001.

Kalamees, R., K. Püssa, K. Zobel, and M. Zobel. 2012. Restoration potential of the persistent soil seed bank in successional calcareous (alvar) grasslands in Estonia. Applied Vegetation Science 15:208-218. https://doi.org/10.1111/j.1654109X.2011.01169.x.

Kassahun, A., H. A. Snyman, and G. N. Smit. 2009. Soil seed bank evaluation along a degradation gradient in arid rangelands of the Somali region, eastern Ethiopia. Agriculture, Ecosystems and Environment 129:428-436. https: //doi.org/10.1016/j.agee.2008.10.016.

Leder, C. V., G. Peter, F. A. Funk, and D. V. Pelaez. 2017. Consequences of anthropogenic disturbances on soil seed bank diversity and nurse shrub effect in a semiarid rangeland. Biodiversity Conservation 26:2327-2346. https: //doi.org/10.1007/s10531-017-1358-0.

Lonsdale, W. M. 1999. Global Patterns of Plant Invasions and the Concept of Invasibility. Ecology 80(5):1522-1536. https://doi.org/10.1890/0012-9658(1999)080[1522:GPOPIA]2.0.CO;2.

Magurran, A. E. 1988. Ecological diversity and its measurement. Princeton University Press, New Jersey. Pp. 179. https: //doi.org/10.1007/978-94-015-7358-0.

Marañón, T. 1995. Ecología de los bancos de semilla en el suelo: una revisión de estudios españoles. Revista Pastos 25(1):3-25.

Márquez, S., G. Funez, M. Cabido, and E. Pucheta. 2002. Efectos del pastoreo sobre el banco de semillas germinable y la vegetación establecida en pastizales de montaña del centro de Argentina. Revista Chilena de Historia Natural 75: 327-337. https://doi.org/10.4067/S0716-078X2002000200006.

Martin, M., E. F. A. Morici, and H. Petruzzi. 2018. Efecto del tiempo de pastoreo sobre el banco de semillas y los parámetros estructurales de Piptochaetium napostaense. Semiárida. Revista de la Facultad de Agronomía UNLPam 2: 9-15. https://doi.org/10.19137/semiarida.2018(02).9-15.

McCune, B., and M. J. Mefford. 2011. PC-ORD. Multivariate Analysis of Ecological Data. Version 6. MjM Software, Gleneden Beach, Oregon, U.S.A.

Montoya, E., C. E. Suárez, E. Morici, and H. D. Estelrich. 2017. Banco de semillas en un pastizal intervenido de La Pampa: datos preliminares en el marco de su restauración. XXXVI Jornadas Argentinas de Botánica, XXVIII Reunión Anual de la Sociedad de Botánica de Chile y II Reunión Científica de la Asociación Micológica Carlos Spegazzini. 52:231.

Morici, E., V. Doménech García, G. Gómez Castro, A. Kin, A. M. Saenz, and C. M. Rabotnikof. 2009. Diferencias estructurales entre parches de pastizal del caldenal y su influencia sobre el banco de semillas, en la provincia de La Pampa, Argentina. Agrociencia 43:529-537.

Mueller-Dombois, D., and H. Ellemberg.1974. Aims and methods of vegetation ecology. Wiley International (eds.).

Naito, A. T., and D. M. Cairns. 2011. Patterns and processes of global shrub expansion. Progress in Physical Geography 35(4):423-442. https://doi.org/10.1177/0309133311403538.

Noellemeyer, E., A. R. Quiroga, and D. Estelrich. 2006. Soil quality in three range soils of the semi-arid Pampa of Argentina. Journal of Arid Environments 65:142-155. https://doi.org/10.1016/j.jaridenv.2005.07.007. 
Piudo, M. J., and R. Y. Cavero. 2005. Banco de semillas: comparación de metodologías de extracción, de densidad y de profundidad de muestreo. Publicaciones de Biología. Universidad de Navarra. Serie Botánica 16:71-85.

Quiroga, E., L. Blanco, and E. Orionte. 2009. Evaluación de estrategias de rehabilitación de pastizales áridos. Ecología Austral 19:107-117.

Rauber, R., D. Steinaker, M. Demaría, and D. Arroyo. 2014. Factores asociados a la invasión de pajas en bosques de la región semiárida central argentina. Ecología Austral 24:320-326. https://doi.org/10.25260/EA.14.24.3.0.9.

Raup, D. M., and R. E. Crick. 1979. Measurement of faunal similarity in paleontology. Journal of Paleontology 53: 1213-1227.

Roberts, H. A. 1981. Seed banks in soils. Advances in Applied Biology 6:1-55.

Rodriguez-Salazar, M. E., S. Álvarez-Hernández and E. Bravo-Núñez. 2001. Coeficientes de asociación. Pp. $34-35$ en Plaza y Valdéz (eds.). México.

Rúgolo de Agrazar, Z. E., P. E. Steibel, and H.O. Troiani. 2005. Manual ilustrado de las gramíneas de la provincia de La Pampa. (Ed. Universidad Nacional de La Pampa y Universidad Nacional de Río Cuarto. Córdoba).

Sokal, R. R., and F. J. Rohlf. 1981. Biometry. The principles and practice of statistics in biological research. W. H. Freemna and Company. New York.

Somovilla Lumbreras, D., R. Páez, E. G. Jobbágy, and M. D. Nosetto. 2019. Cambios en el contenido de carbono orgánico del suelo tras el rolado de bosques secos en San Luis (Argentina). Ecología Austral 29:112-119. https: //doi.org/10.25260/EA.19.29.1.0.815.

Soriano, A. 1990. Missing strategies for water capture in the Patagonian semi-desert. Academia Nacional de Ciencias Exactas, Físicas y Naturales 5:135-139.

Suárez, C. E., M. G. Ronco, and H. D. Estelrich. 2010. Distribución y Estructura de una Población de Heterotheca subaxillaris (Lam.) Britt and Rusby (falso alcanfor) en la provincia de La Pampa, Argentina. Revista de la Facultad de Agronomía. UNLPam 21:19-30.

Suárez, C. E., and D. Estelrich. 2018. Heterotheca subaxillaris (Lam.). Pp. 435-448 en O. A. Fernández, E. S. Leguizamón and M. Acciaresi (eds.). Malezas e Invasoras de la Argentina. Historia y Biología. Tomo III.

Suárez, C. E., H. D. Estelrich, E. A. F. Morici, R. D. Ernst, N. Sawczuk, M. Pérez Payeras, et al. 2018. Evaluación de la vegetación en renovales de Prosopis caldenia intervenidos con distintas técnicas de manejo. Pp. 294-305 en V. Rusch, G. Caballé, S. Varela and J. P. Diez (eds.). Actas. IV Congreso Nacional de Sistemas Silvopastoriles. Ediciones INTA.

Suárez, C. E., E. Montoya, E. Morici, H. D. Estelrich, and R. D. Ernst. 2019. Intervenciones en el caldenal: ¿Favorece el ingreso de las especies exóticas? XXXVII Jornadas Argentinas de Botánica. Boletín de la Sociedad Argentina de Botánica 54:159-160.

Taguchi, Y. H., and Y. Oono. 2005. Relational patterns of gene expression via non-metric multidimensional scaling analysis. Bioinformatics 21:730-740. https://doi.org/10.1093/bioinformatics/bti067.

Templeton, A. R., and D. A. Levin. 1979. Evolutionary consequences of seed pools. American Naturalist 114:232-249. https://doi.org/10.1086/283471.

Thompson, K., and J. P. Grime. 1979. Seasonal variation in seed banks of herbaceous species in ten contrasting habitats. Journal of Ecology 67:893-921. https://doi.org/10.2307/2259220.

Vázquez, P., E. Adema, and S. Aimar. 2013. Dinámica de la fenología de la vegetación a partir de series temporales de NDVI de largo plazo en la provincia de La Pampa. Ecología Austral 23:77-86. https://doi.org/10.25260/ EA.13.23.2.0.1163.

Wang, N., J. Y. Jiao, Y. F. Jia, W.J. Bai, and Z. G. Zhang. 2010. Germinable soil seed bank sand the restoration potential of abandoned cropland on the Chinese Hilly-Gullied Loes Plateaux. Environmental Management 46:367-377. https: //doi.org/10.1007/s00267-010-9535-X.

Wang, Y., D. Jiang, O. Toshio, and Q. Zhou. 2013. Recent Advances in Soil Seed Bank Research. Contemporary Problems of Ecology 6(5):520-524. https://doi.org/10.1134/S1995425513050181. 